\title{
Optimal balance of efficacy and tolerability of oral triptans and telcagepant: a review and a clinical comment
}

\author{
Peer Tfelt-Hansen
}

Received: 23 December 2010/ Accepted: 28 January 2011/Published online: 25 February 2011

(C) The Author(s) 2011. This article is published with open access at Springerlink.com

\begin{abstract}
Dose-response curves for headaches relief and adverse events (AEs) are presented for five triptans: sumatriptan, zolmitriptan, naratriptan, almotriptan, and frovatriptan, and the CGRP antagonist telcagepant. The upper part of the efficacy curve of the triptans is generally flat, the socalled ceiling effect; and none of the oral triptans, even in high doses, are as effective as subcutaneous sumatriptan, In contrast, AEs increases with increasing dose without a ceiling effect. The optimal dose for the triptans is mainly determined by tolerability. Telcagepant has an excellent tolerability and can be used in migraine patients with cardiovascular co-morbidity. Based on the literature the triptans and telcagepant are rated in a table for efficacy and tolerability.
\end{abstract}

Keywords Migraine - Acute treatment - Triptans · Dose-response curve $\cdot$ Telcagepant

We conclude that a single $6 \mathrm{mg}$ dose of sumatriptan given subcutaneously is a highly effective, rapid-acting, and welltolerated treatment for migraine attacks. [1]

\section{Introduction}

The vignette suggests that "the philosophers's stone" has been found with the introduction of sumatriptan.

\section{P. Tfelt-Hansen $(\square)$}

Department of Neurology, Danish Headache Center,

University of Copenhagen, Glostrup Hospital,

Glostrup, Denmark

e-mail: ptha@glo.regionh.dk
Subcutaneous sumatriptan $6 \mathrm{mg}$ and subcutaneous naratriptan $10 \mathrm{mg}$ are both highly effective drugs. Headache relief at $2 \mathrm{~h}$ was 81, 85-89\% [1-4], and 91\% [2], respectively; but in both cases there is a high incidence of adverse events (AEs) (53-71, 85\% [2, 3]). Most of these AEs after subcutaneous sumatriptan were reported as being minor and transient in one study [1] whereas in another simultaneously conducted study $20 \%$ of the AEs after sumatriptan and $17 \%$ after placebo were described as severe [2].

In clinical practice with oral triptans not all migraine patients respond to a triptan and AEs can be a problem. The optimal balance of efficacy and tolerability depends on the combined dose-response curves for both antimigraine effect and incidence of AEs. These dose-response curves for oral triptans will be reviewed, the findings discussed and finally my clinical comments will be presented.

\section{Methods and results}

Dose-defining, randomised, controlled trials (RCTs) of triptans were searched for in PubMed and in The Headaches [5]. Studies defining the dose-response curves of oral triptans for both efficacy and the incidence of $\mathrm{AE}$ were selected for analysis. In addition, large dose-defining studies on the CGRP antagonist telcegepant were searched for.

For three triptans (zolmitriptan, naratriptan, and almotriptan) the balance of efficacy and tolerability could be evaluated by drawing the curves from one dose-defining study as shown in Figs. 2, 3, and 4. Two dose-defining studies $[5,6]$ were needed to evaluate the full doseresponses curves for sumatriptan and frovatriptan (Figs. 1, 2, and 6). For rizatriptan and eletriptan the incidence of 


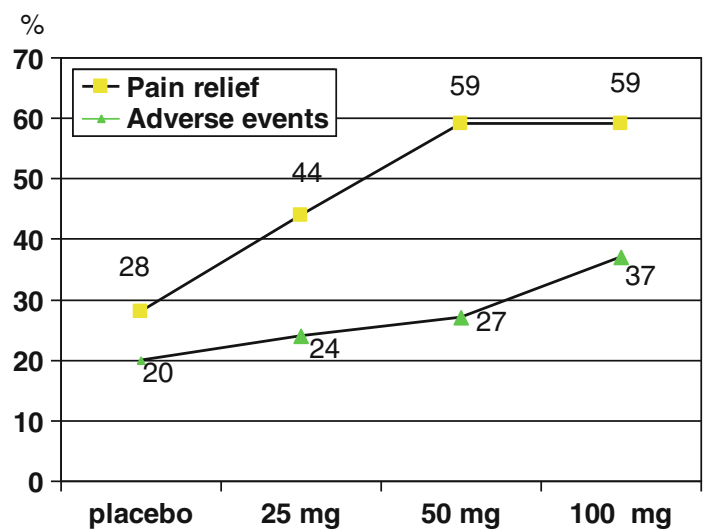

Fig. 1 Effect of sumatriptan 25, 50, and $100 \mathrm{mg}$ on headache relief and adverse events in one RCT [6]

AEs was not presented [7-11] and only the results for efficacy of these two triptans are mentioned briefly.

Sumatriptan is the first and standard triptan and it took two studies, from 1991 and 1998, before the dose-response curve for oral sumatriptan could be established (Figs. 1, 2) $[6,12]$. It is evident from Figs. 1 and 2 that there is an upper flat part of the dose-response curve for efficacy, starting at sumatriptan $50 \mathrm{mg}$, and there is no increase in efficacy up to the $300 \mathrm{mg}$ dose. The incidence of AEs increases with increasing dose of sumatriptan, reaching a maximum of $53 \%$ after $300 \mathrm{mg}$ sumatriptan. $25 \mathrm{mg}$ sumatriptan was the minimum effective dose [6]. For sumatriptan $50 \mathrm{mg}$ there was $7 \%$ more AEs than after placebo (Fig. 1a) which is quite similar to the $9 \%$ found in one meta-analysis [13]. The recommended starting dose of oral sumatriptan is $50 \mathrm{mg}$. This choice is based on maximal efficacy and reasonable tolerability (Figs. 1, 2).

The dose-response curves for zolmitriptan are shown in Fig. 3 [14]. Again there is a flat upper part for efficacy. The starting dose for this plateau is $2.5 \mathrm{mg}$ zolmitriptan. The AEs increase with increasing dose and reach a maximum of

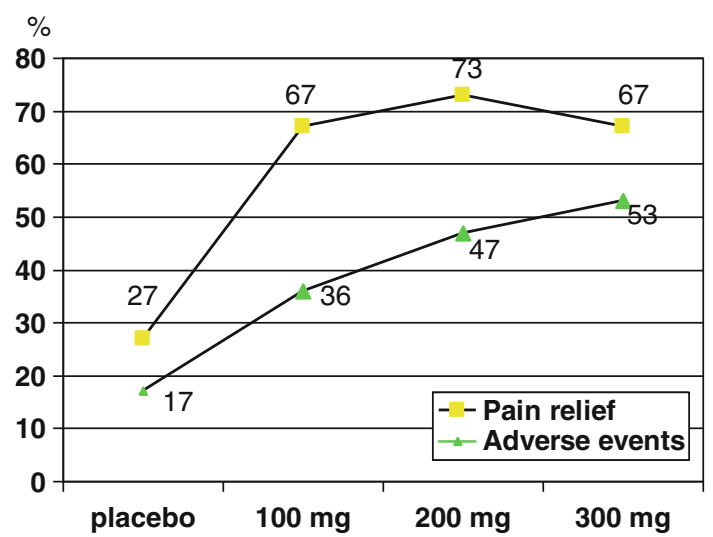

Fig. 2 Effect of sumatriptan 100, 200, and $300 \mathrm{mg}$ on headache relief and adverse events in one RCT [7]

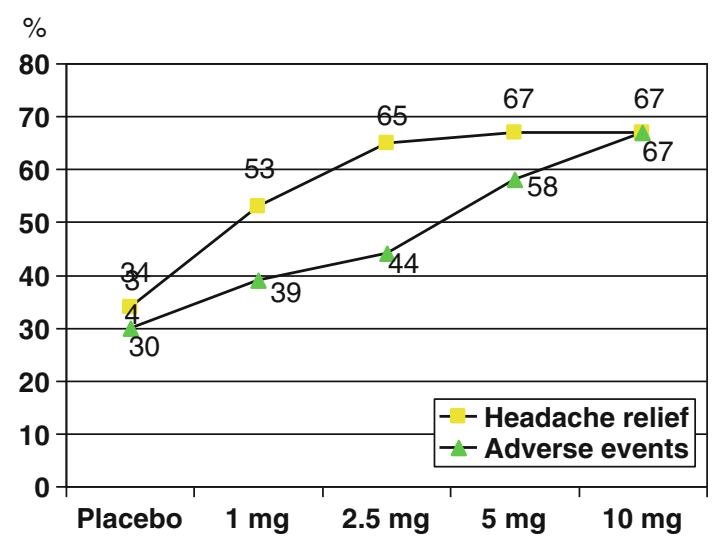

Fig. 3 Effect of zolmitriptan 1, 2.5, 5, and $10 \mathrm{mg}$ on headache relief and adverse events in one RCT [14]

$67 \%$ after $10 \mathrm{mg}$ zolmitriptan. For zolmitriptan $2.5 \mathrm{mg}$ there were $14 \%$ more AEs than after placebo. This incidence is quite similar to the $15 \%$ found in a meta-analysis [13]. The biggest difference between efficacy and AEs (Fig. 2) was observed at the $2.5 \mathrm{mg}$ dose which is therefore the recommended dose for zolmitriptan [15].

Oral naratriptan apparently has a dose-response curve for efficacy [16] with a plateau which starts at $7.5 \mathrm{mg}$ (Fig. 4). For AEs there is a similar plateau in this dose range. At $2.5 \mathrm{mg}$ there are no more AEs than with placebo, as has also been observed in a meta-analysis [13]. The $2.5 \mathrm{mg}$ dose of naratriptan was subsequently chosen as a recommended dose without any more AEs than placebo, the so-called "gentle triptan" [17].

The dose-response curves for almotriptan are shown in Fig. 5 [18] and there is a slight increase in efficacy from $6.25(56 \%)$ to $25 \mathrm{mg}(66 \%)$. The incidences of AEs are remarkably low and first at $25 \mathrm{mg}$ there is a slight increase compared with placebo. The AEs up to $12.5 \mathrm{mg}$ (16-18\%) were described as being mild in the majority of patients whereas the AEs after $25 \mathrm{mg}(25 \%)$ were described as

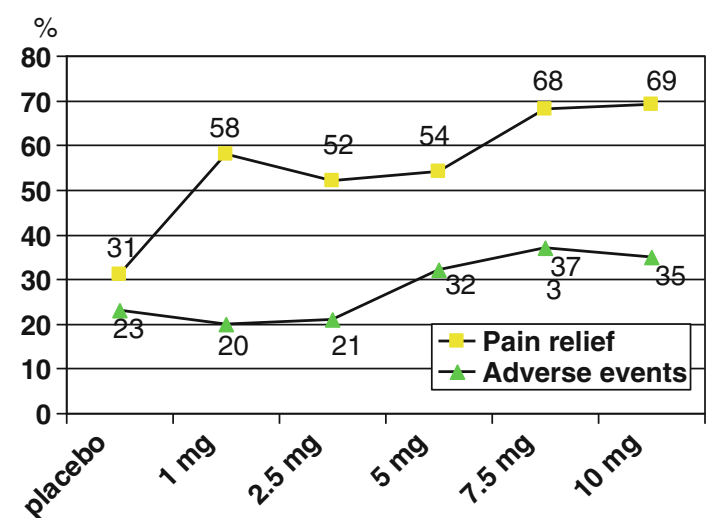

Fig. 4 Effect of naratriptan 1, 2.5, 5, 7.5, and $10 \mathrm{mg}$ on headache relief and adverse events in one RCT [16] 


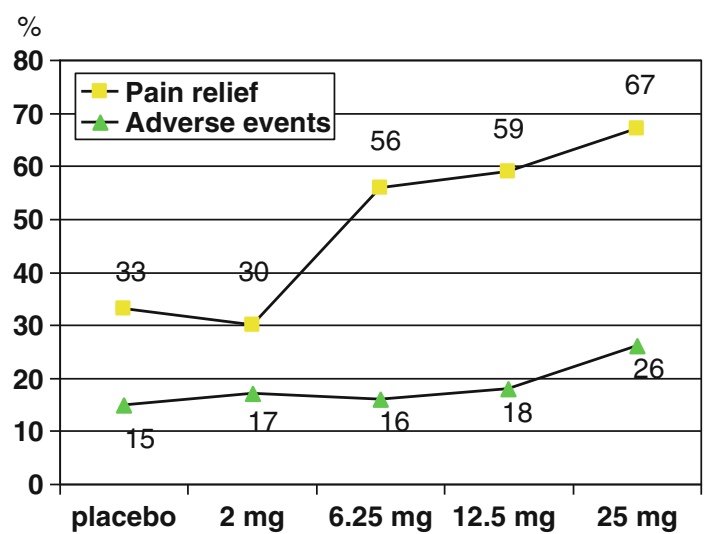

Fig. 5 Effect of almotriptan 2, 6.25, 12.5, and $25 \mathrm{mg}$ on headache relief and adverse events in one RCT [18]

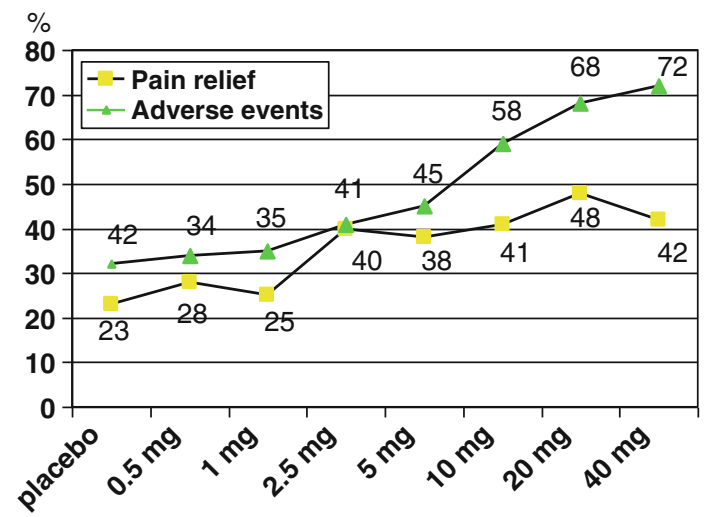

Fig. 6 Effect of frovatriptan $0.5,1,2.5,5,10,20$, and $40 \mathrm{mg}$ on headache relief and adverse events in two RCTs [19]

being of moderate intensity in $48 \%$ of cases. Also in a meta-analysis almotriptan $12.5 \mathrm{mg}$ was found to have AEs at the placebo level [13]. Mostly based on the change in intensity of AEs almotriptan $12.5 \mathrm{mg}$ was chosen as the recommended dose $[15,18]$.

The efficacy of frovatriptan was evaluated by pooling the results of two RCTs [19]. The combined results are shown in Fig. 6. From $2.5 \mathrm{mg}$ and with higher doses there is a flat dose-response curve. Below $2.5 \mathrm{mg}$ there is no efficacy. The incidences of AEs increase with dose and there is a maximum of $72 \%$ at $40 \mathrm{mg}$. The recommended dose is frovatriptan $2.5 \mathrm{mg}$, the lowest dose with efficacy.

For rizatriptan and eletriptan the total incidences of AEs (any patients with an $\mathrm{AE}$ ) are not reported but the incidences of individual AEs are given in tables [8-11]. Thus only the dose-response curves for efficacy of these two triptans can be evaluated. In one dose-finding RCT $(n=417)$ headache relief was $18 \%$ with placebo, and 21 , 45 , and $48 \%$, with rizatriptan doses of $2.5,5$, and $10 \mathrm{mg}$, respectively [10]. In a RCT $(n=449)$ exploring the upper part of the dose-response curve for rizatriptan headache relief was $18 \%$ with placebo and 52, 56, and $67 \%$ with 10 ,

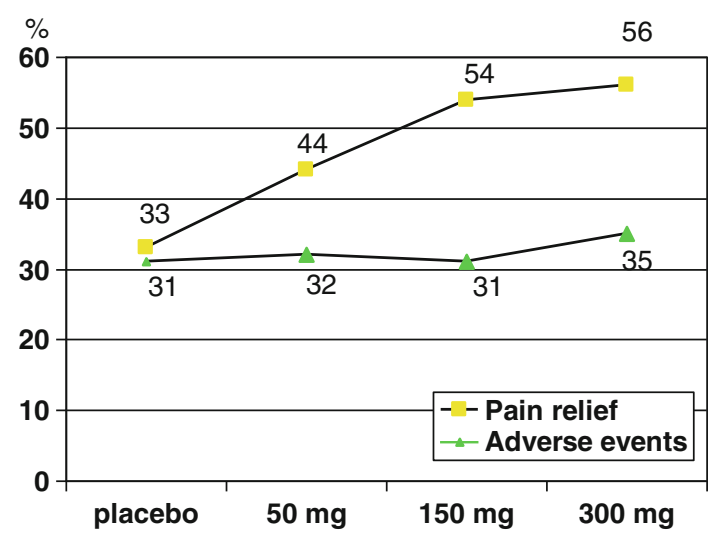

Fig. 7 Effect of telcagepant 50, 150, and $300 \mathrm{mg}$ on headache relief and adverse events in one RCT [21]

20, and $40 \mathrm{mg}$ doses of rizatriptan. AEs occurred more frequently after a $40 \mathrm{mg}$ dose of rizatriptan [11]. In one RCT $(n=1,190)$ investigating the effect of eletriptan headache relief was $20 \%$ with placebo an 47,62 , and $59 \%$ with 20, 40, and $80 \mathrm{mg}$ doses of eletriptan [9] and in another RCT $(n=1334)$ [8] headache relief was $22 \%$ with placebo and 47,62, and 59\% with the eletriptan doses of 20, 40, and $80 \mathrm{mg}$, respectively. In both RCTs AEs were comparable for eletriptan $20 \mathrm{mg}$ and placebo $[8,9]$. AEs from different trial programmes are difficult to compare because of differences in the methodology of collecting AEs. In a meta-analyses any AE (placebo-subtracted) were 7 and $13 \%$ after 5 and $10 \mathrm{mg}$ doses of rizatriptan; and 2, 6, and $18 \%$ after 20,40 , and $80 \mathrm{mg}$, respectively, doses of eletriptan [13]. There is thus also for these two triptans an increase in the incidence of AEs with increase in doses.

Telcagepant, a calcitonin gene-related peptide (CGRP) receptor antagonist, is currently being developed for the acute treatment of migraine. In one small dose-defining RCT [20] doses of 300 and $600 \mathrm{mg}$ telcagepant were found comparable and the $300 \mathrm{mg}$ dose was selected for further investigation. The dose-response curves for telcagepant in doses from 50 to $300 \mathrm{mg}$ are shown in Fig. 7 [21]. The incidence of AEs is at the placebo level, confirming the lack of CGRP antagonists on human vasculature [22], and there is probably a plateau for efficacy from 150 or $300 \mathrm{mg}$ and further up [21, 23]. The recommended dose will probably be $300 \mathrm{mg}$ telcagepant, a dose with maximum effect and AEs on placebo level.

\section{Discussion}

In 2002, it was stated that triptans have served as the foot soldiers or the advances in migraine research during the latter part of the twentieth century [24]. How effective are these revolutionary drugs then in clinical practice? 
The triptans are per se highly effective drugs confer the 85-91\% headache relief at $2 \mathrm{~h}$ after subcutaneous sumatriptan and naratriptan [1-3]. Theoretically, it should be possible by increasing the oral dose of a triptan to obtain similar high response rates. This is, however, not the case. Even with similar plasma concentrations of the sumatriptan and naratriptan after oral and subcutaneous administration the injection is still superior to the oral form [4]. As shown in Figs. 1, 2, 3, and 5 there is for several triptans, sumatriptan, zolmitriptan, and frovatriptan, a flat upper part of the dose-response curves. In addition, the efficacy even with very high doses, e.g., the $40 \mathrm{mg}$ dose of frovatriptan. $(42 \%)$ and of rizatriptan $(67 \%)$, is not near the efficacy of the subcutaneous form, vide supra. This higher efficacy of injected triptans compared with the oral form is most likely due to a quicker rise in blood concentrations after subcutaneous injections [4].

The upper part of the dose-effect curves for several triptans, sumatriptan, zolmitriptan, and frovatriptan (Figs. 1, 2, 3 , and 6) demonstrate a ceiling effect for response on migraine pain. This ceiling effect is especially pronounced for frovatriptan for which a 16-fold increase to $40 \mathrm{mg}$ from the $2.5 \mathrm{mg}$ dose did not result in an increase in efficacy (see Fig. 6). In contrast the dose-response curves for AEs show that the incidence of AEs increases with increasing doses (Figs. 1, 2, 3, 4, 5, and 6), and there is no indications of a ceiling effect.

Only reporting the incidence of AEs does not in all cases give the full picture of the clinical impact of the AEs. Thus for almotriptan $12.5 \mathrm{mg}$ AEs were reported as mild whereas for $25 \mathrm{mg}$ they were reported as moderate [18]. The global impact of AEs should be measured on suitable quality of life scales in the future [25].

Compared to the traditionally used drug, ergotamine, which in addition to its 5-HT1B/1D has agonistic effect on e.g., the dopamine $\mathrm{D}_{2}$ receptor [26], the triptans act selectively on the 5-HT1B/1D receptor [15, 27] and should thus have a better tolerability profile than ergotamine. Thus in one RCT rectal ergotamine $2 \mathrm{mg}(73 \%)$ was slightly superior to rectal sumatriptan $25 \mathrm{mg}$ (63\%) for headache relief but caused significantly more nausea and/or vomiting: 28 and 7\%, respectively [15, 28].

Even if just recording the incidence of AEs in the balance between efficacy and tolerability is not the ideal measure of tolerability it is fair measure for the potential for AEs of a triptan in the migraine population and in several cases the incidence of AEs has determined the recommended doses of the triptans. The recommended doses are in most cases a realistic compromise between efficacy and tolerability.

The new CGRP antagonist telcagepant has an excellent tolerability with AEs on the placebo level (see Fig. 6 [21, 23]). Telcagepant has a headache relief of $56 \%$ and has a $26 \%$ pain-free response [29] which is lower than $40 \%$ for rizatriptan $10 \mathrm{mg}$ [13].

\section{Clinical comments}

My personal rating of the triptans and telcagepant is given in Table 1. It is based both on comparative RCTs [5], two systematic reviews [27, 30], and a meta-analysis [13]. For efficacy + is given for a drug somewhat better than placebo, ++ is given for an effective drug, and +++ for a highly effective drug. For tolerability 0 is given for no more AES than placebo, + for $<10 \%$ more AEs than placebo, ++ for $<25 \%$ more AEs than placebo, and +++ for $>25 \%$ more AEs than placebo.

It should be noted that there are most likely inter-individual difference to responses to triptans. Thus one patient A may use one triptan successfully whereas patient B may prefer another triptan. This variability among triptans is most likely due to both a pharmacokinetic and pharmacodynamic variability among the drugs [31]. From a pharmacokinetic point of view almotriptan has the advantage of a high oral bioavailability of $80 \%$ and is more unlikely to
Table 1 Efficacy and tolerability of triptans and telcagepant

For explanation of $(+$ to +++$)$ for efficacy and of ( 0 to +++$)$ for AES potential, see text. The rating is based on $[13,15,21$, $23,27,30]$

\begin{tabular}{lll}
\hline $\begin{array}{l}\text { Drug and dose } \\
(\mathrm{mg})\end{array}$ & $\begin{array}{l}\text { Efficacy } \\
(+,++, \text { and }+++)\end{array}$ & $\begin{array}{l}\text { Adverse events potential } \\
(0,+,++, \text { and }+++)\end{array}$ \\
\hline Subcutaneous sumatriptan: 6 & +++ & +++ \\
Sumatriptan: 50 & ++ & + \\
Sumatriptan: 100 & ++ & ++ \\
Naratriptan: 2.5 & + & 0 \\
Rizatriptan: 10 & ++ & ++ \\
Zolmitriptan: 2.5 & ++ & ++ \\
Eletriptan: 40 & ++ & ++ \\
Almotriptan: 12.5 & ++ & 0 \\
Frovatriptan: 2.5 & + & + \\
Telcagepant: 300 & ++ & 0 \\
\hline
\end{tabular}


Table 2 Incidence, most common AEs, chest-related AEs in placebo-controlled RCTs after oral administration of zolmitriptan $2.5 \mathrm{mg}[15,33]$

\begin{tabular}{lcc}
\hline & $\begin{array}{l}\text { Placebo }(n=401) \\
(\%)\end{array}$ & $\begin{array}{l}\text { Zolmitriptan 2.5 mg } \\
(n=498)(\%)\end{array}$ \\
\hline $\begin{array}{l}\text { Patients with at least one AE } \\
\text { Most common AEs }\end{array}$ & $117(29)$ & $227(46)$ \\
Asthenia & $13(3)$ & $16(3)$ \\
Dry mouth & $7(2)$ & $16(3)$ \\
Nausea & $15(4)$ & $45(9)$ \\
Dizziness & $16(4)$ & $42(8)$ \\
Somnolence & $12(3)$ & $30(6)$ \\
Paresthesia & $6(1)$ & $21(4)$ \\
Warm sensitation & $7(2)$ & $21(4)$ \\
Chest-related AEs & & $13(3)$ \\
Chest tightness & $2(<1)$ & $1(1<1)$ \\
Chest pain & $1(<1)$ & $1(<1)$ \\
Chest heaviness & $0(0)$ & $1(<1)$ \\
Chest pressure & $1(<1)$ & \\
\hline
\end{tabular}

vary among subjects than e.g., sumatriptan with an oral bioavailability of $14 \%[15,27]$. Because of no more AEs in RCTs than placebo (see Fig. 5) almotriptan $12.5 \mathrm{mg}$ can apparently (see Table 1) be a first choice triptan if no AEs are tolerated. It should be noted, however, that some patients can experience so-called "triptan" symptoms (see below) even after almotriptan as after other triptans. Sumatriptan is now of patent in most countries and sumatriptan 50-100 mg should therefore in clinical practice be the triptan of first choice when triptans are used de novo in migraine patients.

Even if the AEs after triptans are in most cases mild to moderate and transient they can be frightening for the patients which should be informed about possible AEs. Somnolence and asthenia are reported as AEs of triptan but they are most likely partly treatment-emergent CNS symptoms of the migraine attack following the treatment with triptans [26]. Even so they are experienced by the patients as bothersome AEs. The so-called "triptans" symptoms [32] are shown for placebo and $2.5 \mathrm{mg}$ recommended dose of zolmiriptan in Table $2[15,33]$. Note that zolmitriptan $2.5 \mathrm{mg}$ caused $17 \%$ more adverse events than placebo. Chest symptoms (mainly tightness and pressure) have been reported to occur in up to $20 \%$ (tablets) and $40 \%$ (subcutaneous injection) of the patients treated with sumatriptan some time [15, 34]. Such symptoms can be a frightening experience for the patients, and they should be warned in advance of the risk of the symptoms and should be informed about the transient and generally benign nature.

If telcagepant becomes available it will be the drug of first choice for the patients with migraine and cardiovascular diseases or high risk for such diseases. It will also be a good choice if the migraine patient has intolerable AEs when treating with triptans.
It should be noted that with any drug used in acute migraine treatment there is a different balance of efficacy and tolerability in the individual patient and there is thus no standard dose that suites every patient. In addition, some patients may prefer a very effective drug with some AEs to a drug with lower efficacy and virtually no AEs. Drugs and doses should thus be tailored to the need of the individual patient.

Finally, it is important to note that the majority of the patients experience no AEs with use of the oral specific $5 \mathrm{HT} 1 \mathrm{~B} / 1 \mathrm{D}$ receptor agonists, the triptans, in the recommended doses (see, Fig 1, 2, 3, 4, and 5).

When AEs occur they are in most cases mild to moderate and transient. On balance, the triptans with their proven efficacy and an acceptable tolerability profile have been a major step forward in the acute treatment of migraine.

\section{Conflict of interest None.}

Open Access This article is distributed under the terms of the Creative Commons Attribution License which permits any use, distribution and reproduction in any medium, provided the original author(s) and source are credited.

\section{References}

1. The Subcutaneous Sumatriptan International Study Group (1991) Treament of migraine attacks with sumatriptan. N Eng J Med 325:316-321

2. Cady RK, Wendt JK, Kirchner JR, Sargent JD, Rothrock JF, Skaggs H Jr (1991) Treatment of acute migraine with subcutaneous sumtriptan. JAMA 265:2831-2835

3. Dahlöf C, Hogenhuis L, Olesen J, Petit H, Ribbat J, Schoenen J et al (1998) Early clinical experience with subcutaneous 
naratriptan in the acute treatment of migraine: a dose-ranging study. Eur J Neurol 5:469-477

4. Tfelt-Hansen P (2007) Parenteral versus oral sumatriptan and naratriptan: plasma levels and efficacy in migraine. A comment. J Headache Pain 8:273-276

5. Olesen J, Goadsby PJ, Ramadan N, Tfelt-Hansen P, Welch KMA (2006) The Headaches, 3rd edn edn. Lippincott Williams and Wilkins, Philadelphia, pp 1-1169

6. Pfaffenrath V, Cunin G, Sjonell G, Pendergast S (1998) Efficacy and safety of sumatriptan tablets $(25 \mathrm{mg}, 50 \mathrm{mg}$, and $100 \mathrm{mg}$ ) in the acute treatment of migraine: defining the optimum doses of oral sumatriptan. Headache 38:184-190

7. Tfelt-Hansen P, Teall J, Rodriguez F, Giacovazzo M, Paz J, Malbecq W, Block GA, Reines SA, Visser WH (1998) Oral rizatriptan versus oral sumatriptan: a direct comparative study in the acute treatment of migraine. Rizatriptan 030 study Group. Headache 38:748-755

8. Sheftell F, Ryan R, Pitman V, Eletriptan Steering Committee (2003) Efficacy, safety and tolerability of oral eletriptan for treatment of acute migraine: a multicenter, double-blind, placebocontrolled study conducted in the United States. Headache 43:202-213

9. Stark R, Dahlöf C, Haughie S, Hettiarchchi J, Eletriptan Steering Committee (2002) Efficacy, safety and tolerability of oral eletriptan in the acute treatment of migraine: results of a phase III, multicentre, placebo-controlled study across three attacks. Cephalalgia 22:23-32

10. Gijsmant H, Kramer MS, Sargent J, Tuchman M, Maczura-Wolfe D, Polis A et al (1997) Double-blind, placebo-controlled, dosefinding study of rizatriptan (MK-462) in the acute treatment of migraine. Cephalalgia 17:647-651

11. Visser WH, Terwindt GM, Reines SA, Jiang K, Lines CR, Ferrari Md (1996) Rizatriptan vs sumatriptan in the acute treatment of migraine. A placebo-controlled, dose-ranging study. Dutch/US Rizatriptan Study Group. Arch Neurol 53:1132-1137

12. Sumatriptan - an oral dose-defining study. The Oral Sumatriptan Dose-Defining Study Group (1991) Eur Neurol 31: 300-305

13. Ferrari MD, Roon KI, Lipton RB, Goadsby PJ (2001) Oral triptans (serotonin 5-HT (1B/1D) agonists in the acute migraine treatment: a metaanalysis of 53 triptans. Lancet 358:1668-1675

14. Rapoport AM, Ramadan NM, Adelman JU, Mathew NT, Elkind AH, Kudrow DB et al (1997) Optimizing the dose of zolmiriptan (Zomig, 311C90) for the acue reatment of migraine. A multicenter, double-blind, placebo-controlled, dose range-finding study. Neurology 49:1210-1218

15. Saxena PR, Tfelt-Hansen P (2006) Triptans, 5HT1B/1D agonists in the acute treatment of migraine. In: Olesen J, Goadsby PJ, Ramadan NM, Tfelt-Hansen P, Welch KMA (eds) The Headaches, 3rd edn edn. Lippincott Williams and Wilkins, Philadelphia, pp 469-503

16. Havanka H, Dahlöf C, Pop PH, Diener HC, Winter P, Whitehouse $\mathrm{H}$ et al (2000) Efficacy of naratriptan tablets in the acute treatment of migraine: a dose-ranging study. Naratriptan S2WB2004 Study Group. Clin Ther 22:970-980

17. Salonen R (2001) Naratripan: the gentle triptan. In: Humphrey P, Ferrari M, Olesen J (eds) The triptans: novel drugs for migraine. Oxford University Press, Oxford, pp 228-235

18. Dahlöf C, Tfelt-Hansen P, Massiou H, Fazekas A, Almotriptan Study Group (2001) Dose finding, placebo-controlled study of oral almotriptan in the acute treatment of migraine. Neurology 57:1811-1817

19. Rapoport A, Ryan R, Goldstein J, Keywood C (2002) Dose range-finding studies with frovatriptan in the acute treatment of migraine. Headache 42(Suppl 2):S74-S83

20. Ho TW, Mannix LK, Fan X, Assaid C, Furtek C, Jones CJ et al (2008) Randomized controlled trial pf an oral CGRP receptor antagonist. MK-0974, in acute treatment of migraine. Neurology 70:1304-1312

21. Connor KM, Shapiro RE, Diener H-C, Lucas S, Kost J, Fan X et al (2009) Randomized, controlled trial of telcagepant for the acute treatment of migraine. Neurology 73:970-977

22. Petersen KA, Birk S, Lassen LH, Kruuse C, Jonassen O, Lesko L, Olesen J (2005) The CGRP-antagonist BIBN4096BS does not affect cerebral or systemic haemodynamics in healthy volunteers. Cephalalgia 25:139-147

23. Ho TW, Ferrari MD, Dodick DW, Galet J, Kost J, Fan X et al (2008) Efficacy and tolerability of MK-0974 (telcagepant), a new oral antagonist of calcitonin gene-related peptide receptor, compared with zolmitriptan for acute migraine: a randomized, placebo-controlled, parallel-treatment trial. Lancet 372:2115-2123

24. Goadsby PJ (2002) New directions in migraine research. J Clin Neurosci 9:368-373

25. Tfelt-Hansen P, Bjarnason NH, Dahlöf CGH, Derry S, Loder E, Massiou H, Task Force on Adverse Events in Migraine Trials of the Subcommittee on Clinical Trials of the International Headache Society (2008) Evaluation and registration of adverse events in clinical drug trials in migraine. Cephalalgia 28:683-688

26. Goadsby PJ, Dodick D, Almas M, Diener H-C, Tfelt-Hansen P, Lipton RB, Parsson B (2007) Treatment emergent CNS symptoms following triptan therapy are part of the migraine attack. Cephalalgia 27:254-262

27. Tfelt-Hansen P, Saxena PR (2006) Ergot alkaloids in the acute treatment of migraine. In: Olesen J, Goadsby PJ, Ramadan NM, Tfelt-Hansen P, Welch KMA (eds) The Headaches, 3rd edn edn. Lippincott Williams and Wilkins, Philadelphia, pp 459-467

28. Tfelt-Hansen P (2009) Unpublished sumatriptan clinical trials. Lancet 374:1501-1502

29. Tfelt-Hansen P (2011) Excellent tolerability but relatively low initial efficacy of the oral CGRP antagonist telcagepant in the treatment of migraine. Headache 51:118-123

30. Pascual J, Mateos V, Roig C, Sanchez-Del-Rio M, Jiménez D (2007) Marketed triptans in the acute treatment of migraine: a systematic review on efficacy and tolerability. Headache 47:1152-1168

31. Tfelt-Hansen P, Edvinsson L (2007) Pharmacokinetic and pharmacodynamic variability as possible causes for different drug responses in migraine. A comment. Cephalalgia 232.7:10911093

32. Mathew NT (1997) Serotonin1D (5-HT1D) agonists and other agents in acute migraine. Neurol Clin 15:61-83

33. Edmeads JG, Millson DS (1997) Tolerability profile of zolmitriptan (ZomigTM, 311C90, a novel dual central and peripherally acting $5 \mathrm{HT} 1 \mathrm{~B} / 1 \mathrm{D}$ agonist. International experiemce based on $>3000$ subjects treated with zolmitriptan. Cephalalgia 17:41-52

34. Dahlöf CG, Saiers J (1998) Sumatriptan injection and tablets in clinical practice: results of a survey of 707 migraineuers. Headache 38:756-763 